\title{
Monitoria de Alergia e Imunologia Clínica durante o ensino remoto em tempo de pandemia: Um relato de experiência
}

\author{
Teaching Assistantship of Allergy and Clinical Immunology during remote teaching while in a \\ pandemic: An experience report \\ Ayudantía Docente de Alergia e Inmunología Clínica durante la enseñanza a distancia en tiempos \\ de pandemia: Un informe de experiencia
}

\section{Resumo}

A monitoria consiste em uma atividade extracurricular que visa despertar o interesse de discentes pela prática da docência e pesquisa científica. Nesse sentido, o presente artigo consiste em um relato de experiência da monitoria de uma disciplina eletiva da Faculdade de Medicina da Universidade Federal de Alagoas, frente aos obstáculos criados pelo cenário pandêmico devido à disseminação do novo coronavírus. O propósito deste relato, além de compartilhar a vivência dos monitores, é retratar a relevância da monitoria da disciplina de Alergia e Imunologia Clínica para a formação acadêmica dos estudantes de medicina, mesmo em face de um contexto de aulas remotas. As atividades de monitoria ocorreram durante o segundo semestre de 2020 e o primeiro semestre de 2021, por meio de atividades síncronas e assíncronas em ambientes virtuais. Nessa perspectiva, ainda que de forma não tradicional da qual costumava ser desenvolvida, a monitoria foi capaz de promover a cooperação entre discentes e docentes, estimulando o compartilhamento e a troca de conhecimentos, além de ter permitido a superação de situações de adversidades, o aprimoramento e adaptação das estratégias de ensino para o formato digital e o aprendizado na graduação, especificamente na área da alergia e imunologia clínica.

Palavras-chave: Ensino; Educação em saúde; Monitoria; Ensino online. 


\begin{abstract}
The teaching assistantship consists of an extracurricular activity that aims to awaken the interest of students in the practice of teaching and scientific research. In this sense, the present article consists of an experience report of the tutoring experience in an elective course at the Faculty of Medicine of the Federal University of Alagoas through the pandemic scenario caused by the dissemination of the new coronavirus. The purpose of this report, in addition to sharing the experience of the teaching assistants, is to depict the relevance of tutoring for the education of medical students enrolled in the Allergy and Clinical Immunology course, despite the context of remote learning. Tutoring activities took place during the second half of 2020 and the first half of 2021, through synchronous and asynchronous activities in virtual environments. From this perspective, although in a non-traditional way than what it used to be presented, tutoring was able to promote cooperation between teachers and different students, encouraging them to exchange knowledge, in addition to having allowed the overcoming of adversity situations, improvement and adaptation of teaching strategies to a digital format and promoting a better learning experience at graduation, specifically in the area of allergy and clinical immunology.
\end{abstract}

Keywords: Teaching; Health education; Teaching Assistantship; Online education.

\title{
Resumen
}

La ayudantía docente consiste en una actividad extraescolar que tiene como objetivo despertar el interés de los estudiantes por la práctica de la docencia y la investigación científica. En este sentido, el presente artículo consiste en un relato vivencial de la experiencia de tutoría en un curso electivo en la Facultad de Medicina de la Universidad Federal de Alagoas a través del escenario pandémico provocado por la diseminación del nuevo coronavirus. El propósito de este informe, además de compartir la experiencia de los profesores asistentes, es dar a conocer la relevancia de la tutoría para la formación de los estudiantes de medicina matriculados en el curso de Alergia e Inmunología Clínica, a pesar del contexto de aprendizaje a distancia. Las actividades de tutoría se llevaron a cabo durante el segundo semestre de 2020 y el primer semestre de 2021, a través de actividades sincrónicas y asincrónicas en entornos virtuales. Desde esta perspectiva, aunque de una manera no tradicional a la que solía presentarse, la tutoría supo promover la cooperación entre docentes y diferentes alumnos, incentivando el intercambio de conocimientos, además de haber permitido la superación de situaciones de adversidad, mejora y adaptación de estrategias de enseñanza a formato digital y promoción de una mejor experiencia de aprendizaje en la graduación, específicamente en el área de alergia e inmunología clínica.

Palabras clave: Enseñanza; Educación para la salud; Ayudantía Docente; Educación en línea.

\section{Introdução}

A monitoria acadêmica é um ato presente nas Instituições de Ensino Superior (IES) previsto no artigo 84 da Lei $\mathrm{N}^{\circ}$ 9384, responsável por estabelecer as diretrizes e bases da educação nacional. Tal artigo, refere que "os discentes da educação superior poderão ser aproveitados em tarefas de ensino e pesquisa pelas respectivas instituições, exercendo funções de monitoria, de acordo com seu rendimento e seu plano de estudos" (Lei n. 9394, 1996).

De acordo com Câmara, Akaishi, Cabreira e Camargo (1997), a monitoria acadêmica possui alguns objetivos dos quais podem ser citados: potencialização de aprendizagem; utilização de nível apropriado de linguagem; redução da ansiedade negativa frente ao corpo docente; possibilidade de trabalhar em grupos menores; integração entre docentes e discentes; participação do processo de avaliação e, ainda, incentivo à pesquisa e extensão.

Como uma preparação para a docência, há, na atividade de monitoria, o desenvolvimento da didática, que, no contexto da educação, é vista por Costa et al. (2020) como um elemento essencial que permite a realização de uma reflexão profunda a respeito de sua prática pedagógica. Souza \& do Espírito Santo (2013) observou que uma prática didática que possibilita somar, inovar e trazer suporte técnico para desempenhar a tarefa de mediador não é fácil, porém, caso o profissional não se ajuste às novas demandas do processo de ensino e aprendizagem, tenderá a ser rotulado como mau profissional.

O monitor é considerado um agente do processo ensino-aprendizagem, capaz de intensificar a relação professor-alunoinstituição (Natário, 2007). Nesse sentido, de acordo com Natário e Santos (2010), um dos deveres do monitor é "reunir-se com o docente para juntos elaborarem um plano de trabalho, considerando percepções, ideias, observações sobre os alunos e sobre a instituição, realizando encaminhamentos concretos que vão desde a adequação dos objetivos propostos pelo programa de ensino até a avaliação das condições de realização da programação, a preparação de aulas, a checagem dos procedimentos, estratégias e avaliações, além de outras questões que possibilitem discutir e providenciar ações que favoreçam o ensino e a aprendizagem". 
Com o avanço da COVID-19, foi decretado, pelo Diretor-Geral da OMS, estado de pandemia em 11/03/2020 (Novelli et al., 2020). Dentre as diversas medidas utilizadas pelos países para mitigar a disseminação do vírus, temos como exemplo o auto-isolamento ou quarentena (Bedford et al., 2020). Desse modo, de acordo com Silva et al. (2020), os ambientes de educação remotos vêm aumentando em razão da pandemia do COVID-19 que interrompeu as aulas presenciais em todas as instituições de ensino no país.

Assim, o presente artigo tem como objetivo relatar a experiência de acadêmicos do curso de medicina da Universidade Federal de Alagoas enquanto monitores da disciplina de Alergia e Imunologia Clínica.

\section{Metodologia}

Estudo de natureza descritiva, com abordagem qualitativa, do tipo relato de experiência, o qual é um tipo de estudo em que prevalece a abordagem empírica, crítica e reflexiva acerca da experiência vivenciada pelos pesquisadores, exigindo, portanto, respaldo científico (Vasconcellos, Maia, \& Andrade, 2018).

Este relato foi construído a partir das experiências vivenciadas pelos monitores da disciplina eletiva "Alergia e Imunologia Clínica", ofertada à graduação de medicina da Faculdade de Medicina (FAMED) da Universidade Federal de Alagoas (UFAL) para discentes a partir do terceiro semestre da graduação.

A monitoria em questão acontecia, originalmente, de forma presencial, mas, devido à situação de Pandemia do vírus Sars-Cov-2, ocorreu de forma remota, com aulas síncronas (Ao vivo) e assíncronas (Gravações de aulas ao vivo disponibilizadas na plataforma YouTube), durante o segundo semestre de 2020, no que compreendeu o Período Letivo Excepcional (PLE) e o primeiro semestre de 2021, correspondente ao Semestre 2020.1 da graduação.

Dessa forma, foi realizada revisão e análise minuciosa das atividades realizadas pelos professores e monitores ao longo da disciplina, de modo a compartilhar as mudanças e novas experiências que se adequaram ao novo contexto de monitoria.

\section{Resultados e Discussão}

\section{Contexto da Monitoria}

A disciplina alvo da monitoria faz parte das disciplinas eletivas que são ofertadas aos discentes do curso de graduação em Medicina pela Universidade Federal de Alagoas. Em decorrência da vigente pandemia deflagrada pela COVID-19, a disciplina foi ofertada de maneira remota durante o PLE, contando com uma carga horária total de 30 horas. As atividades síncronas ocorriam em horário fixo, às quintas-feiras, das 16:00h até às 17:30h. Já as atividades assíncronas, compostas por estudos dirigidos e questionários dos assuntos abordados em aula, podiam ser realizadas pela turma no decorrer da semana.

Neste contexto, durante o PLE e o Período 2020.1, as aulas foram ministradas remotamente pelo Ambiente Virtual de Aprendizagem (AVA), pertencente ao sistema institucional da Universidade, vinculado à plataforma Moodle e ao GSuite, que integra ferramentas como o Google meet, e que facilitava os encontros e reuniões virtuais, e o Google Classroom, que permitia uma melhor organização das atividades e do conteúdo programático da disciplina. Ambas as ferramentas possibilitaram uma comunicação facilitada, bem como o acesso e o registro das atividades planejadas. O aplicativo de mensagens instantâneas Whatsapp também foi utilizado com o propósito de facilitar a comunicação entre os professores, monitores e alunos matriculados na disciplina, além de permitir uma melhor divulgação de materiais de apoio e demais conteúdos de interesse para a disciplina. Deste modo, para a devida realização das atividades, acompanhamento das discussões e presença nas aulas e encontros síncronos, fez-se necessária a disponibilidade de conexão com a internet.

De modo geral, as aulas teóricas foram desenvolvidas em torno de duas unidades, onde os conteúdos teóricos foram divididos e apresentados de modo expositivo com o auxílio de ferramentas como o PowerPoint e vídeos inseridos no YouTube no canal próprio da monitoria, que eram projetados durante as aulas síncronas realizadas pelo Google Meet. Nestes encontros, 
sempre estavam presentes ao menos um professor da disciplina e os monitores. Após os encontros síncronos, atividades e estudos dirigidos eram adicionados no Google Classroom, de forma que os alunos pudessem aprofundar e complementar o conteúdo previamente visto de forma assíncrona no decorrer da semana.

Assim sendo, o objetivo principal da disciplina de Alergia e Imunologia Clínica foi abordar os conteúdos de relevância clínica que envolvem o escopo da disciplina e que constituem importante base teórica para o médico generalista.

\section{Experiência e Discussão}

As atividades desenvolvidas pelos monitores compreenderam desde o acompanhamento e assistência aos professores durante as aulas, incluindo a administração do Google Classroom até o desenvolvimento de materiais didáticos.

As atividades realizadas podem ser divididas em: acompanhamento, assistência e gravação das aulas síncronas, upload das aulas no youtube; postagem das aulas no Google Classroom; criação de formulários de fixação de conhecimento e postagem no Google Classroom; criação e divulgação de materiais didáticos e assistência aos alunos.

Além disso, foi criado um grupo com os acadêmicos matriculados na disciplina, acadêmicos monitores e professores do WhatsApp, a fim de se promover mais um espaço para sanar questionamentos referentes aos conteúdos da disciplina.

Historicamente, de acordo com Melo et al. (2014), o ensino na saúde tem sido pautado no uso de métodos tradicionais no qual o professor é colocado no centro do processo educativo como transmissor de conteúdos, sendo o aluno um mero expectador, passivo e repetidor. A criação de materiais didáticos, criação de formulários centrados em casos clínicos reais e relacionados com a prática clínica e a interação livre e sem distinção hierárquica proporcionada pelas redes sociais, buscavam transpassar esse problema educacional.

A monitoria desenvolvida consistiu em 12 horas semanais que foram divididas em dois dias distintos, independente dos monitores estarem sempre dispostos para sanar dúvidas e auxiliar os alunos e professores com comunicação através do grupo do Whatsapp. Desse modo, um momento com seis horas de duração, foi realizado com a presença das monitoras nas salas virtuais, a fim de dar suporte a docente, realizando sua gravação e fazendo edições (caso necessárias), postando no canal do Youtube, e em seguida disponibilizando-a no Google Classroom; Além disso, outro horário foi destinado para o momento elaboração de materiais complementares, criação de formulários de fixação de conhecimento e postagem no Google Classroom; criação e divulgação de materiais didáticos, além da correção desses formulário, totalizando, assim, as seis horas.

Os formulários de fixação foram desenvolvidos a partir de casos clínicos, que são situações reais ou fictícias, com dados resumidos e intencionalidade cognitiva, cujo objetivo é introduzir ou ilustrar determinado conhecimento (Hokama, Hokama, \& Batista, 2018). Cada caso clínico era composto por perguntas que requisitavam o desenvolvimento de respostas dissertativas pelos alunos acerca a situação apresentada, assim, o processo para se chegar a ela por meio da análise e discussão individual e coletiva das informações expostas no caso, de acordo com Vieira, Vieira, e Pasqualli (2017), promove o raciocínio crítico e argumentativo dos estudantes.

Em virtude da pandemia, houve a necessidade de utilizar o meio virtual como ambiente de aprendizagem, sendo essencial para a continuidade das atividades científicas e educacionais. O século XXI foi marcado pelas mudanças socioculturais influenciadas principalmente pelas tecnologias digitais da informação e comunicação (TDIC) que estão cada vez mais presentes em quase todas as camadas da sociedade (Silva, 2017) e essas tecnologias foram peça-chave para redução de prejuízos, ao adotar plataformas digitais de ensino e integração acadêmica foi possível alcançar os objetos de aprendizagem. O papel dos monitores, em coadunar e auxiliar na resolução das necessidades de discentes e docentes foi outro ponto indispensável (Goudouris, Giannella, \& Struchiner, 2013). Quanto mais um professor domina diferentes tecnologias digitais e consegue contextualizá-las para o ensino, de acordo com Souza e Carvalho (2021), mais diversificado será o seu trabalho, algo que, consequentemente, poderá implicar positivamente na aprendizagem dos alunos. 
Diante da impossibilidade de encontros presenciais, o ambiente virtual demonstrou-se um grande aliado inevitável para a disseminação e fixação do aprendizado. Entretanto, existem limitações diante do ensino remoto, como a necessidade capacitação dos profissionais envolvidos, os problemas de conectividades ou técnicos dos participantes e a dificuldade de concentração diante de telas virtuais. Mas a possibilidade das mesmas aulas nas formas síncrona e assíncrona, além de gerar um maior contato entre discentes e docentes, possibilitou que cada aluno pudesse visualizar quantas vezes achar necessário, e em horários e ritmos próprios. Essa é uma das vantagens que, segundo Azevedo et al (2020), a internet trouxe, possibilitando o maior aproveitamento da disciplina a partir do momento em que o aluno pode acessar o conteúdo no momento em que estiver mais à vontade $\mathrm{O}$ grande acervo de imagens e vídeos apresentados pelos professores, durante as aulas ministradas, foi altamente efetivo para a construção do aprendizado, aliado aos formulários semanais, que garantiram uma maior fixação dos temas abordados (da Silva, Neto, \& dos Santos, 2020).

O ensino de alergologia e imunologia clínica, é fundamental na formação do médico generalista, trata-se de uma disciplina clínica e laboratorial, atuando no estudo, diagnóstico e tratamento das desordens imunológicas. Além disso, diante da prevalência crescente de doenças alérgicas mundialmente, que podem vir a ser até mesmo emergências médicas, como nos quadros de anafilaxia, condição que é inclusive desconhecida pelos médicos não especialistas, o que dificulta o diagnóstico e o manejo. É necessário o processo de educação em alergologia e imunologia clínica, a fim de melhorar a condução dos quadros alérgicos e imunológicos. (Moreira, Mundim, \& de Jesus, 2018)

A monitoria é de extrema importância para o auxílio dos alunos e professores da disciplina, ajudando no alcance dos objetivos de aprendizagem e facilitando o acesso ao conhecimento e tecnologias para tal. Nesse ambiente ocorre a iniciação à docência, criando um ambiente ativo de ensino-aprendizagem. Além disso, torna possível ao monitor uma melhor construção/consolidação do conhecimento da disciplina anteriormente concluída, mediante: intermediação das dúvidas sobre alergologia e imunologia clínica entre discentes-docentes, revisão de assuntos para produção científica, correção dos formulários das atividades semanais. Outro grande benefício da monitoria, que impacta positivamente na vida acadêmica e até mesmo na futura vida profissional, é a ampliação do "networking social". (Santos \& Batista, 2015).

\section{Considerações Finais}

A participação na monitoria contribui para o crescimento dos monitores no âmbito acadêmico, pois é através dela que o monitor é estimulado a aprofundar seus conhecimentos na disciplina além de que o monitor se torna um ator essencial para o desenvolvimento da disciplina de forma remota.

A realização da monitoria estimula também ao acadêmico monitor o senso de responsabilidade e de cooperação tanto com os outros monitores quanto com os docentes, o impulsiona na atualização frente às tecnologias que podem melhorar o aproveitamento da disciplina.

A satisfação ao ver o crescimento dos acadêmicos após a contribuição pedagógica, ainda que num contexto desafiante imposta pela pandemia, incentiva ainda mais a busca por melhorias na disciplina, além de ser uma grande oportunidade para o despertar do interesse pela docência.

Nesse sentido, considera-se importante a realização de novos trabalhos científicos sobre o tema, permitindo uma melhor compreensão sobre o desempenho e desenvolvimento de atividades extracurriculares, em especial a monitoria, no contexto de ensino remoto. Além disso, sugere-se a elaboração de pesquisas científicas que avaliem o impacto de novas estratégias de ensino e de comunicação com os alunos em ambiente virtual, levando em consideração não só a experiência de docentes e monitores, como também dos próprios discentes, de maneira a avaliar a assimilação de conteúdos abordados nos ambientes virtuais de aprendizagem. 


\section{Referências}

Azevedo, C. R. L. de, Farias, M. E. L. de, \& Bezerra, C. C. (2020). Monitoria acadêmica em uma disciplina semipresencial: Relato de experiência. Research, Society and Development, 9(4), e39942788-e39942788. https://doi.org/10.33448/rsd-v9i4.2788

Bedford, J., Enria, D., Giesecke, J., Heymann, D. L., Ihekweazu, C., Kobinger, G., Lane, H. C., Memish, Z., Oh, M., Sall, A. A., Schuchat, A., Ungchusak, K., \& Wieler, L. H. (2020). COVID-19: Towards controlling of a pandemic. The Lancet, 395(10229), 1015-1018. https://doi.org/10.1016/S0140-6736(20)30673-5

Câmara, S. S. P., Akaishi, P. M. S., Cabreira, M. a. S., \& Camargo, M. C. B. A. (2020). Monitoria Acadêmica em Semiologia Médica: Descrição e Avaliação de uma Nova Experiência. Revista Brasileira de Educação Médica, 21, 47-54. https://doi.org/10.1590/1981-5271v21.1-008

da Silva, E., Neto, J., \& dos Santos, M. (2020). Pedagogia da pandemia: reflexões sobre a educação em tempos de isolamento social. Revista Latino-americana de Estudos Científicos, 1 (4). http://dx.doi.org/10.46375/relaec.31695 .

Costa, K. M. R. da, Lino, M. R. B., Miranda, C. E. S., Borges, J. W. P., Bandeira, H. M. M., \& Lopes, M. do S. L. (2020). Didática e docência multiprofissional em saúde: Relato de experiência. Research, Society and Development, 9(2), e45921984-e45921984. https://doi.org/10.33448/rsd-v9i2.1984

Goudouris, E. S., Giannella, T. R., \& Struchiner, M. (2013). Tecnologias de informação e comunicação e ensino semipresencial na educação médica. Revista Brasileira de Educação Médica, 37, 396-407. https://doi.org/10.1590/S0100-55022013000300012

Hokama, P. O. M., Hokama, N. K., \& Batista, N. (2018). Caso Motivador como Estratégia Problematizadora e Integradora no Ensino Médico em um Curso de Oncologia. Revista Brasileira de Educação Médica, 42(4), 165-174. https://doi.org/10.1590/1981-52712015v42n4rb20170080

Lei n. 9.394, de 20 de dezembro de 1996. (1996). Estabelece as diretrizes e bases da educação nacional. Diário Oficial [da] República Federativa do Brasil. Brasília, DF, 23/12/1996, p.27.833

Melo, M. C. de, Queluci, G. de C., \& Gouvêa, M. V. (2014). Problematizando a residência multiprofissional em oncologia: Protocolo de ensino prático na perspectiva de residentes de enfermagem*. Revista da Escola de Enfermagem da USP, 48, 706-714. https://doi.org/10.1590/S0080-623420140000400019

Moreira, I. F., de Oliveira Mundim, A. C., \& de Jesus, K. P. (2018). Ensino de alergia e imunologia clínica em dois cursos de graduação em medicina de universidades públicas em Maceió/AL. Gep News, 1(1), 87-92.

Natário, E. G. (2007). Monitoria: um espaço de valorização docente e discente. Anais do $3^{\circ}$ Seminário Internacional de Educação do Guarujá, 2007 (1, 29). Santos: Editora e Gráfica do Litoral.

Natário, E. G., \& Santos, A. A. A. dos. (2010). Programa de monitores para o ensino superior. Estudos de Psicologia (Campinas), 27, 355-364. https://doi.org/10.1590/S0103-166X2010000300007

Novelli, G., Biancolella, M., Mehrian-Shai, R., Erickson, C., Godri Pollitt, K. J., Vasiliou, V., Watt, J., \& Reichardt, J. K. V. (2020). COVID-19 update: The first 6 months of the pandemic. Human Genomics, 14(1), 48. https://doi.org/10.1186/s40246-020-00298-w

Santos, G. M., \& Batista, S. H. S. da S. (2015). Academic monitoring in health teaching: challenges and possibilities in an interprofessional health curriculum. ABCS Health Sciences, 40(3). https://doi.org/10.7322/abcshs.v40i3.796

Silva, D. dos S., Andrade, L. A. P., \& Santos, S. M. P. dos. (2020). Alternativas de ensino em tempo de pandemia. Research, Society and Development, 9(9), e424997177. https://doi.org/10.33448/rsd-v9i9.7177

Silva, J. B. da. (2017). O Contributo Das Tecnologias Digitais Para O Ensino Híbrido: O Rompimento Das Fronteiras Espaço-Temporais Historicamente Estabelecidas E Suas Implicações No Ensino. ARTEFACTUM - Revista de estudos em Linguagens e Tecnologia, 15(2), Article 2. http://artefactum.rafrom.com.br/index.php/artefactum/article/view/1531

Souza, J. B. de, \& Carvalho, T. dos S. (2021). Formação de professores para o uso de tecnologias digitais: Um relato de experiência no Colégio Estadual Professora Luzia Carvalho Silva. Research, Society and Development, 10(4), e33710414156-e33710414156. https://doi.org/10.33448/rsd-v10i4.14156

Souza, S. M. V. C., \& do Espírito Santo, E. (2013). Reflexão da Didática como mediadora entre a teoria e prática pedagógica. Universitas Humanas. https://doi.org/10.5102/univhum.v10i1.2154

Vasconcellos, L., Maia, P., \& Andrade, J. (2018). Relato de experiência: o processo de ensinagem do método SOAP. Revista Recien - Revista Científica De Enfermagem, 8(23), 47. https://doi.org/10.24276/rrecien2358-3088.2018.8.23.47-53

Vieira, J. de A., Vieira, M. M. M., \& Pasqualli, R. (2017). Estudo de caso como estratégia de ensino para a Educação Profissional e Tecnológica. Série-Estudos - Periódico do Programa de Pós-Graduação em Educação da UCDB, 143-159. https://doi.org/10.20435/serie-estudos.v22i44.1012 